\title{
An Electron-Microscope Study of Germination of Conidia of Botrytis cinerea
}

\author{
By LILIAN E. HAWKER AND R. J. HENDY \\ Department of Botany, University of Bristol
}

(Received 12 June 1963)

\begin{abstract}
SUMMARY
Conidia of Botrytis cinerea have a 2-layered cell wall, a thin electrondense outer layer and a thicker electron-transparent inner one. The cell contents comprise typical nuclei, mitochondria and endoplasm with a surrounding plasmalemma and a sparse endoplasmic reticulum. There are a few small peripheral vacuoles. On germination the outer spore wall ruptures and the emergent germ tube is surrounded by the elastic inner one and what appears to be a sheath of mucilage. A cross wall with a simple central pore is laid down at the base of the germ tube at a very early stage. As the germ tube elongates the contents of the spore flow into it, causing a large vacuole to form in the centre of the original spore. The numbers of nuclei and mitochondria suggest that divisions of these organelles take place during the early stages of germination. The mucilaginous sheath surrounding the germ tube appears to condense to give a thin electron-dense outer zone to the wall at the base of the germ tube.
\end{abstract}

\section{INTRODUCTION}

Hawker \& Abbott (1963) showed that the mature sporangiospores of species of Rhizopus have a relatively thick single-layered cell wall of structure unlike that of the hyphal wall; after soaking in water for $3 \mathrm{hr}$. a thin inner wall can be seen lining the original cell wall. This inner wall consists of tangentially arranged elements and is similar to the walls of young vegetative hyphae. On germination of the spore the original spore wall breaks and the germ tube emerges surrounded by the elastic newly formed inner wall. If the conidium, the typical asexual spore of the Ascomycetes and Fungi Imperfecti, has evolved from the more primitive multispored sporangium by the reduction of the number of spores to one, one would expect this spore to have initially 2-layered walls. Accordingly conidia of Botrytis cinerea Fr. were fixed, embedded in araldite, sectioned and examined under the electron microscope. The techniques were similar to those described by Hawker \& Abbott (1963) except that the sections were stained with sodium plumbite to enhance contrast and most of them were examined with a Siemens Elmiskop I electron microscope instead of the Phillips E.M. 200 electron microscope used in previous studies.

\section{RESULTS}

\section{Fine structure of conidium}

Spore wall. Conidia taken from a mature culture of Botrytis cinerea and fixed immediately ( $\mathrm{Pl}$. 1, fig. 1) have relatively thick walls $(0.5 \mu)$ consisting of two illdefined layers. The outer thinner layer $(\mathrm{OW})$ is electron-dense; its outer surface is 
rough and fluffy but shows no regular ornamentation of the type seen in sporangiospores of Rhizopus; its inner edge is not sharply demarcated. The thick inner wall (IW) is electron-transparent; no structure can be demonstrated by the methods used except that dark round elements are present in the zone immediately adjoining the outer wall.

Cell contents. (i) A distinct plasmalemma (P, Pl. 1, fig. 1) is present, closely adpressed to the cell wall. Lomasomes (Moore \& McAlear, 1961) are common in the plasmalemmas of longer germ tubes (Lo, Pl. 2, fig. 7). (ii) The endoplasm consists of a homogeneous matrix in which scattered membranes (ER) are present. Cisternae or vesicles are rare. A few very small peripheral stellate vacuoles (SV) are present in some spores. (iii) The nuclei (N) are few, relatively small $(2-2 \cdot 5 \mu)$, and surrounded by the typical 3-layered membrane perforated by pores (NP). (iv) Numerous small mitochondria (M) are present. These are of varied size and shape, commonly oval, rod-shaped (M, Pl. 1, fig. 1 ) or $U$-shaped as seen in section $\left(M_{1}\right.$ and $M_{2}$ in PI. 2, fig. 8). Occasionally ( $\mathbf{M}_{3}$ in Pl. 2, fig. 7) a convoluted mitochondrion occurs resembling those demonstrated in 'dormant' spores of Rhizopus by Hawker \& Abbott (1963).

\section{Stages in germination}

Germination of conidia took place after soaking for $6 \mathrm{hr}$. in water at $20^{\circ}$. Lightmicroscope studies showed that the first visible stage in germination was the swelling of the spores through intake of water and the germ tube then emerged. In the absence of a complete series of ultra-thin sections through a number of spores it was not possible to note increase in spore size in electron micrographs. Early stages in the emergence of the germ tube may, however, be seen. Although it was not possible to be certain that a section had gone through the tip of the germ tube, criteria such as the size of the vacuole developing in the parent spore and the condition of the germ tube wall permit an estimate of the relative ages of the photographed germ tubes.

Plate 1, figs. 2 and 3, and Pl. 2, figs. 4 and 5, give a series of stages in germination; figs. 2 and 3 show very early stages. The outer electron-dense spore wall has ruptured in a manner reminiscent of the spore wall of Rhizopus and the emerging germ tube is surrounded by the extending thicker inner spore wall. Where this was in contact with the surrounding medium a diffuse deposit is seen. This probably represents the mucilaginous layer, known to be present, which serves to stick the Botrytis germ tube to a leaf or other firm surface, before the formation of an appressorium (Blackman \& Welsford, 1916). No large central vacuole has as yet developed in the parent spore, indicating that very little of the original spore contents has passed into the young germ tube. Even at this early stage a cross wall has formed at the base of the germ tube. In Pl. 2, figs. 5-7, serial sections show that this basal septum has a central pore through which cytoplasm and organelles can pass. Plate 2, fig. 4, shows a later stage as indicated by the large central vacuole in the parent spore (shown also in sections through germinated spores; Pl. 2, fig. 8) and the presence of nuclei and numerous mitochondria in the germ tube, which itself contains no vacuoles. The number of nuclei seen (Pl. 2, figs. 4, 5) suggests that one or more nuclear divisions have already taken place. Plate 2, fig. 5, shows a still later stage: a vacuole has developed near the base of the germ tube and the wall enclosing this basal part has become more sharply defined with the development of a thin electron- 
dense outer layer which appears to be the result of condensation of the diffuse mucilaginous sheath. Blackman \& Welsford (1916) figured this as a continuation of the spore wall, but the electron micrographs show that this is not so. The pore in the basal septum (Pl. 2, fig. 6) is clearly visible, with a mitochondrion in process of passing into the germ tube.

\section{DISCUSSION}

The evolution of the conidium is a matter of considerable interest and has been the subject of much speculation. Members of the Mucorales can be placed in a series which shows a decrease in numbers of spores in the sporangium from the multispored sporangium of Mucor, Rhizopus and related forms to species such as Chaetocladium or Cunninghamella in which the 'conidia' are assumed to be single-spored sporangia. The conidium is the prevailing asexual spore type in the remaining groups of the Zygomycetes (Entomophthorales and Zoopagales). In the Oomycetes there is an increasing tendency to germination of the zoosporangium as a single spore by means of a germ tube under relatively dry conditions, culminating in Peronospora which does not form zoospores under any circumstances. If the conidium of the Ascomycetes and Fungi Imperfecti has evolved from the Zygomycete type of sporangium one would expect it to have a double wall laid down at the time of formation of the spore initial. If, however, it has evolved from the Oomycete type of zoosporangium, in which the zoospores are without walls, one would expect the conidium wall to be initially of only one layer. The evidence given in this paper supports the view that the Zygomycetes and Ascomycetes (including the majority of the Fungi Imperfecti) have evolved from a common ancestor. The outer wall of the Botrytis conidium would then be homologous with the sporangial wall of Rhizopus and the inner wall with the initial spore wall of the sporangiospore of Rhizopus. A difference arises in the extension of the inner wall of the conidium of Botrytis to surround the developing germ tube, whereas the spore wall of Rhizopus ruptures and the emergent germ tube is surrounded by a newly formed inner wall which is produced rapidly when the sporangiospore is soaked in water or nutrient liquid. No membrane homologous with this was seen in Botrytis. Such an additional membrane is, however, unnecessary in a spore which has a 2-layered wall from the start, and may well have been lost during evolution. Alternatively the singlelayered wall of the Rhizopus spore may formerly have extended round the germ tube and when this wall became thickened and inelastic the new inner wall may have evolved. The 2-layered wall gives an enhanced chance of resistance to desiccation and other unfavourable environmental conditions. Its possession may justifiably be considered to be a more advanced condition than the possession of a wall of only one layer.

The presence of a septum at the base of the germ tube from an early stage is of interest. Unfortunately, no section of the large number examined showed early stages in the formation of this septum. This is not surprising in view of the known rapidity of septum formation in other fungi (Bracker \& Butler, 1963). The pore in the basal septum is of simple structure and would allow practically unimpeded passage of cytoplasm and organelles from the spore into the germ tube. No complex protecting structures, such as those shown by Moore \& McAlear (1962) in certain Ascomycetes or the more complex ones shown by the same authors and by Bracker 
\& Butler (1963) in certain Basidiomycetes, were observed. Portions of the endoplasmic reticulum were, however, usually aligned parallel to the septum ( $\mathrm{Pl}$. 1, figs. 2, 3). Pores of mature hyphae of Botrytis were not observed and no further septa were present in such young germ tubes as those illustrated. More complex structures may well develop in this species with ageing of the septa. No such basal septum was present in the germ tubes of Rhizopus spores studied by Hawker \& Abbott (1963).

Thanks are due to the Departments of Anatomy and Physics, University of Bristol, for allowing us to use their electron microscopes. We are also indebted to the D.S.I.R. for a grant in aid of this investigation.

\section{REFERENCES}

Blackman, V. H. \& Welsford, E. J. (1916). Studies in the physiology of parasitism. II. Infection by Botrytis cinerea. Ann. Bot., Lond. 30, 389.

Bracker, C. E. \& Butler, E. E. (1963). The ultrastructure and development of septa in hyphae of Rhizoctonia solani. Mycologia, 55, 35.

HAWker, L. E. \& Aввотт, P. McV. (1963). An electron microscope study of maturation and germination of sporangiospores of two species of Rhizopus. J. gen. Microbiol. 32, 295.

Moore, R. T. \& McAlear, J. H. (1961). Fine structure of Mycota. 5. Lomasomespreviously uncharacterized hyphal structures. Mycologia, 53, 194.

Moore, R. T. \& McAlear, J. H. (1962). Fine structure of Mycota. 7. Observations on septa of Ascomycetes and Basidiomycetes. Amer. J. Bot. 49, 86.

\section{EXPLANATION OF PLATES}

\section{Plate 1. Botrytis cinerea}

Fig. 1. Approximately transverse section of an ungerminated conidium showing relatively thin, electron-dense, fluffy outer wall (OW); relatively thicker inner wall (IW) showing scattered dark, round elements in zone adjoining outer wall; plasmalemma $(\mathbf{P})$; nucleus $(\mathbf{N})$ with at least one nuclear pore (NP); mitochondria (M) with entire outlines and clearly defined cristae; a few peripheral stellate vacuoles (SV); and sparse endoplasmic reticulum (ER).

Fig. 2. Approximately median transverse section of a conidium at an early stage of germination. The germ tube (GT) enclosed by the inner wall (IW) has emerged through the outer wall (OW) at $\mathrm{X}-\mathrm{X}$ (note broken edge of outer wall); on contact with the surrounding medium a layer of mucilage (Muc) is deposited. Already a basal septum (S) has been laid down, though a septal pore is not visible in this section. Other lettering as in Fig. 1.

Fig. 3. Slightly oblique section of germinating conidium at a later stage than Fig. 2. Lettering as in Fig. 2.

\section{Plate 2. Botrytis cinerea}

Fig. 4. Longitudinal section of a germinating conidium at a somewhat later stage than Fig. 3. In the original spore a large central vacuole $(V)$ has formed due to passage of endoplasm into the germ tube which now contains a number of nuclei (N), mitochondria $(M)$ and a small vacuole $(V)$. The increasing number of nuclei (at least four in this specimen) suggests that several nuclear divisions have already taken place. Many more mitochondria are now present within the conidium. Fig. 5. Germinated conidia at a still later stage. A very large vacuole is now present in the old spore, and the mucilaginous sheath (Muc) appears to have condensed to give a thin electron-dense layer at the base of the germ tube. Five or more nuclei are present in the spore at the upper left of the figure.

Figs. 6, 7. Serial sections of the basal septum of the spore in the lower left of Fig. 5. A septal pore (SP) is clearly visible, with a mitochondrion in the act of passing through. Lomasomes (Lo) are found in the germ tube but have not been seen within the conidium. The contorted mitochondria ( $\mathbf{M}_{3}$ in Fig. 7 ) resemble those demonstrated in dormant spores of Rhizopus.

Fig. 8. Approximately transverse section of a conidium at a late stage of germination, as shown by the large vacuole (V). Mitochondria $M_{1}$ and $M_{2}$ have an interesting shape which may indicate a stage in their division. 

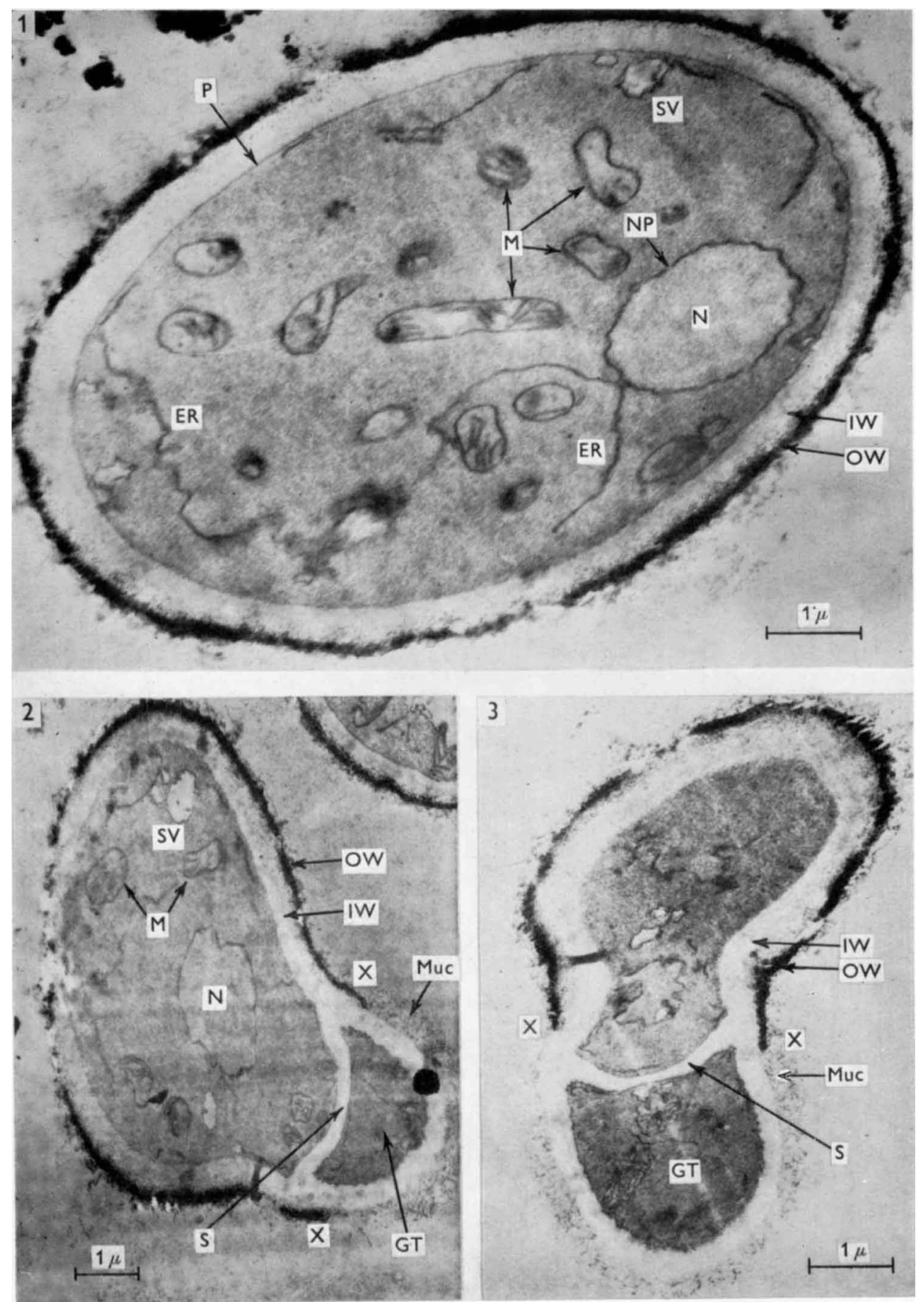

L. E. HAWKER AND R. J. HENDY

(Facing p. 46) 

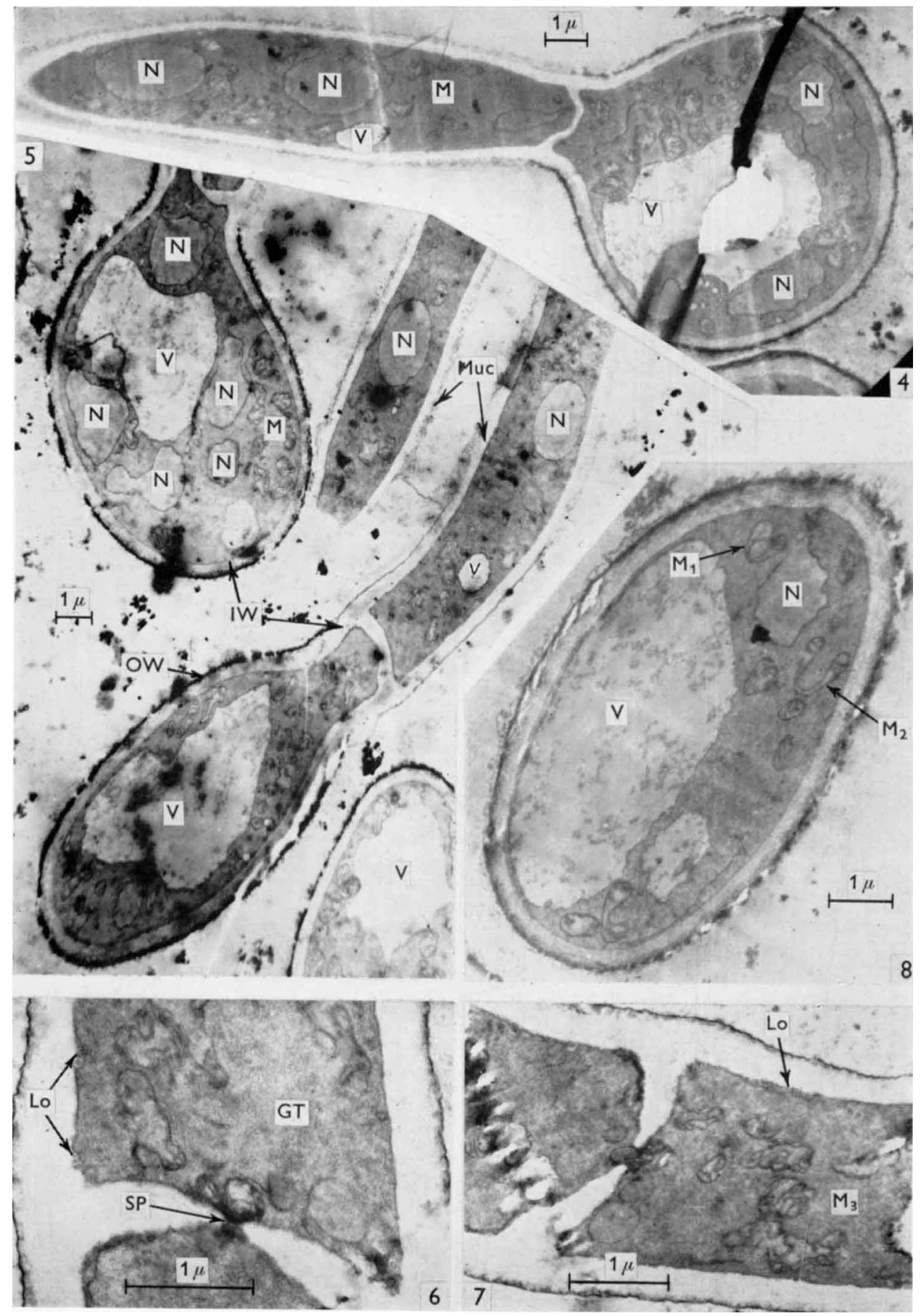

L. E. HAWKER ANI R. J. HENDY 